\title{
EXPANSIONS OF QUADRATIC MAPS IN PRIME FIELDS
}

\author{
MEI-CHU CHANG
}

(Communicated by Bryna Kra)

\begin{abstract}
Let $f(x)=a x^{2}+b x+c \in \mathbb{Z}[x]$ be a quadratic polynomial with $a \not \equiv 0 \bmod p$. Take $z \in \mathbb{F}_{p}$ and let $\mathcal{O}_{z}=\left\{f_{i}(z)\right\}_{i \in \mathbb{Z}^{+}}$be the orbit of $z$ under $f$, where $f_{i}(z)=f\left(f_{i-1}(z)\right)$ and $f_{0}(z)=z$. For $M<\left|\mathcal{O}_{z}\right|$, we study the diameter of the partial orbit $\mathcal{O}_{M}=\left\{z, f(z), f_{2}(z), \ldots, f_{M-1}(z)\right\}$ and prove that there exists $c_{1}>0$ such that

$$
\operatorname{diam} \mathcal{O}_{M} \gtrsim \min \left\{M p^{c_{1}}, \frac{1}{\log p} M^{\frac{4}{5}} p^{\frac{1}{5}}, M^{\frac{1}{13} \log \log M}\right\} .
$$
\end{abstract}

For a complete orbit $\mathcal{C}$, we prove that

$$
\operatorname{diam} \mathcal{C} \gtrsim \min \left\{p^{5 c_{1}}, e^{T / 4}\right\},
$$

where $T$ is the period of the orbit.

\section{INTRODUCTION}

This paper belongs to the general theme of dynamical systems over finite fields. Let $p$ be a prime and $\mathbb{F}_{p}$ the finite field of $p$ elements, represented by the set $\{0,1, \ldots, p-1\}$. Let $f \in \mathbb{F}_{p}[x]$ be a polynomial, which we view as a transformation of $\mathbb{F}_{p}$. Thus if $z \in \mathbb{F}_{p}$ is some element, we consider its orbit

$$
z_{0}=z, z_{n+1}=f\left(z_{n}\right), \quad n=0,1, \ldots,
$$

which eventually becomes periodic. The period $T_{z}=T$ is the smallest integer satisfying

$$
\left\{z_{n}: n=0,1, \ldots, T-1\right\}=\left\{z_{n}: n \in \mathbb{N}\right\} .
$$

We are interested in the metrical properties of orbits and partial orbits. More precisely, for $M<T_{z}$, we define

$$
\operatorname{diam} \mathcal{O}_{M}=\max _{0 \leq n<M}\left|z_{n}-z\right| .
$$

Following the papers [GS] and [CGOS], we study the expansion properties of $f$, in the sense of establishing lower bounds on diam $\mathcal{O}_{M}$. Obviously, if $M \leq T$, then $\operatorname{diam} \mathcal{O}_{M} \geq M$. But, assuming that $f$ is nonlinear and $M=o(p)$, one reasonably expects that the diameter of the partial orbit is much larger. Results along these lines were obtained in GS] under the additional assumption that $M>p^{\frac{1}{2}+\epsilon}$. In this situation, Weil's theorem on exponential sums permits proving equidistribution of the partial orbit. For $M \leq p^{1 / 2}$, Weil's theorem becomes inapplicable and

Received by the editors October 10, 2011 and, in revised form, March 2, 2012.

2010 Mathematics Subject Classification. Primary 11B50, 37A45, 11B75; Secondary 11T23, 37F10, 11G99.

Key words and phrases. Dynamical system, orbits, additive combinatorics, exponential sums. The author's research was partially financed by the National Science Foundation. 
lower bounds on diam $\mathcal{O}_{M}$ based on Vinogradov's theorem were established in CGOS. Our paper is a contribution of this line of research. We restrict ourselves to quadratic polynomials, though certainly the methods can be generalized. (See CCGHSZ for a generalization of Proposition 2 and Theorem 2 to higher degree polynomials and rational maps.)

Our first result is the following.

Theorem 1. There is a constant $c_{1}>0$ such that if $f(x)=a x^{2}+b x+c \in \mathbb{Z}[x]$ with $(a, p)=1$, then with the above notation, for any $z \in \mathbb{F}_{p}$ and $M \leq T_{z}$,

$$
\operatorname{diam} \mathcal{O}_{M} \gtrsim 1 \min \left\{M p^{c_{1}}, \frac{1}{\log p} M^{\frac{4}{5}} p^{\frac{1}{5}}, M^{\frac{1}{13} \log \log M}\right\} \text {. }
$$

In view of Theorem 2, one could at least expect that $\operatorname{diam} \mathcal{O}_{M} \gtrsim \min \left(p^{c}, e^{c M}\right)$ as is the case when $M=T_{z}$.

In the proof, we distinguish the cases $\operatorname{diam} \mathcal{O}_{M}>p^{c_{0}}$ and $\operatorname{diam} \mathcal{O}_{M} \leq p^{c_{0}}$, where $c_{0}>0$ is a suitable constant. First, we again exploit exponential sum techniques (though, from the analytical side, our approach differs from [CGOS and exploits a specific multilinear setup of the problem). More precisely, Proposition 1 in $\S 1$ states that (for $M \leq T$ large enough)

$$
\operatorname{diam} \mathcal{O}_{M} \gtrsim \frac{1}{\log p} \min \left(M^{\frac{5}{4}}, M^{\frac{4}{5}} p^{\frac{1}{5}}\right) .
$$

(Note that (0.5) is a clear improvement over Theorem 8 from [CGOS] for the case $d=2$.)

When diam $\mathcal{O}_{M} \leq p^{c_{0}}$, a different approach becomes available as explained in Proposition 2. In this situation, we are able to replace the $(\bmod p)$ iteration by a similar problem in the field $\mathbb{C}$ of complex numbers, for an appropriate quadratic polynomial $F(z) \in \mathbb{Q}[z]$. Elementary arithmetic permits us to then prove that $\log \operatorname{diam} \mathcal{O}_{M}$ is at least as large as $\frac{1}{13} \log M \log \log M$.

Interestingly, assuming $\mathcal{C}$ is a complete periodic cycle and $\operatorname{diam} \mathcal{C}<p^{c_{0}}$, the transfer argument from Proposition 2 enables us to invoke bounds on the number of rational preperiodic points of a quadratic map, for instance the results from $\mathrm{R}$. Benedetto $[\mathrm{B}$. The conclusion is the following.

Theorem 2. There is a constant $c_{0}>0$ such that if $f(x)=a x^{2}+b x+c \in \mathbb{Z}[x]$ with $(a, p)=1$ and $\mathcal{C} \subset \mathbb{F}_{p}$ is a periodic cycle for $f$ of length $T$, then

$$
\operatorname{diam} \mathcal{C} \gtrsim \min \left\{p^{c_{0}}, e^{T / 4}\right\} .
$$

\section{Diameter of Partial orbits}

Let $f(x)=a x^{2}+b x+c \in \mathbb{Z}[x]$, where $a \not \equiv 0(\bmod p)$. Fix $x_{0} \in \mathbb{F}_{p}$ and denote the orbit of $x_{0}$ by

$$
\mathcal{O}_{x_{0}}=\left\{f_{j}\left(x_{0}\right)\right\}_{j \in \mathbb{Z}^{+}},
$$

where $f_{j}\left(x_{0}\right)=f\left(f_{j-1}\left(x_{0}\right)\right)$ and $f_{0}\left(x_{0}\right)=x_{0}$. The period of the orbit of $x_{0}$ under $f$ is denoted $T=T_{x_{0}}=\left|\mathcal{O}_{x_{0}}\right|$. For $A \subset \mathbb{F}_{p}$, we denote the diameter of $A$ by

$$
\operatorname{diam} A=\max _{x, y \in A} p\left\|\frac{x-y}{p}\right\|,
$$

\footnotetext{
${ }^{1} h \lesssim g$ if there exist constants $C, M$ such that $|h(x)| \leq C g(x)$ for all $x>M$.
} 
where $\|a\|$ is the distance from $a$ to the nearest integer. We are interested in the expansion of part of an orbit.

Proposition 1. For $1 \ll M<T$, consider a partial orbit

$$
\mathcal{O}_{M}=\left\{x_{0}, f\left(x_{0}\right), f_{2}\left(x_{0}\right), \ldots, f_{M-1}\left(x_{0}\right)\right\} .
$$

Then

$$
\operatorname{diam} \mathcal{O}_{M} \gtrsim \frac{1}{\sqrt{\log p}} \min \left(M^{5 / 4}, M^{4 / 5} p^{1 / 5}\right) .
$$

Proof. Let $M_{1}=\operatorname{diam} \mathcal{O}_{M}$. Take $I \subset \mathbb{F}_{p}$ with $|I|=M_{1}$ and $\mathcal{O}_{M} \subset I$; then

$$
|f(I) \cap I| \geq M-1 \text {. }
$$

We will express (1.2) using exponential sums.

Let $0 \leq \varphi \leq 1$ be a smooth function on $\mathbb{F}_{p}$ such that $\varphi=1$ on $I$ and $\operatorname{supp} \varphi \subset \widetilde{I}$, where $\widetilde{I}$ is an interval with the same center and double the length of $I$. Equation (1.2) implies that

$$
\sum_{x \in I} \varphi(f(x)) \geq M
$$

and expanding $\varphi$ in Fourier gives

$$
\varphi(x)=\sum_{\xi \in \mathbb{F}_{p}} \widehat{\varphi}(\xi) e_{p}(x \xi), \text { with } \widehat{\varphi}(\xi)=\frac{1}{p} \sum_{x \in \mathbb{F}_{p}} \varphi(x) e_{p}(-x \xi) .
$$

Combining these gives

$$
\sum_{\xi \in \mathbb{F}_{p}}|\widehat{\varphi}(\xi)|\left|\sum_{x \in I} e_{p}(\xi f(x))\right| \gtrsim M .
$$

We will estimate $\sum_{x \in I} e_{p}(\xi f(x))$ using van der Corput-Weyl.

Take $M_{0}=O(M)$, e.g. $M_{0}=\frac{1}{100} M$. Then

$$
\begin{aligned}
\left|\sum_{x \in I} e_{p}(\xi f(x))\right| & \leq \frac{1}{M_{0}} \sum_{0 \leq y<M_{0}}\left|\sum_{x \in I} e_{p}\left(\xi\left(a(x+y)^{2}+b(x+y)\right)\right)\right|+O\left(M_{0}\right) \\
& \leq \frac{1}{\sqrt{M_{0}}}\left[\sum_{0 \leq y<M_{0}}\left|\sum_{x \in I} e_{p}\left(\xi\left(a x^{2}+2 a x y+b x\right)\right)\right|^{2}\right]^{1 / 2}+O\left(M_{0}\right) \\
& =\frac{1}{\sqrt{M_{0}}}\left|\sum_{\substack{0 \leq y<M_{0} \\
x_{1}, x_{2} \in I}} e_{p}\left(\xi\left(x_{1}-x_{2}\right)\left(a\left(x_{1}+x_{2}\right)+2 a y+b\right)\right)\right|^{1 / 2}+O\left(M_{0}\right) .
\end{aligned}
$$

(The second inequality is by Cauchy-Schwarz.)

Take $\varphi$ sufficiently smooth so as to ensure that

$$
\sum_{\xi \in \mathbb{F}_{p}}|\widehat{\varphi}(\xi)|=O(1)
$$

Equations (1.3) and (1.4) imply

$$
\sum_{\xi \in \mathbb{F}_{p}}|\widehat{\varphi}(\xi)|\left|\sum_{\substack{0 \leq y<M_{0} \\ x_{1}, x_{2} \in I}} e_{p}\left(\xi\left(x_{1}-x_{2}\right)\left(a\left(x_{1}+x_{2}\right)+2 a y+b\right)\right)\right|^{1 / 2} \gtrsim M^{3 / 2}
$$


Hence by Cauchy-Schwarz and (1.5),

$$
\sum_{\xi \in \mathbb{F}_{p}}|\widehat{\varphi}(\xi)| \sum_{\substack{0 \leq y<M_{0} \\ x_{1}, x_{2} \in I}} e_{p}\left(\xi\left(x_{1}-x_{2}\right)\left(a\left(x_{1}+x_{2}\right)+2 a y+b\right)\right) \mid \gtrsim M^{3} .
$$

Fix $x_{1}+x_{2}=s \leq 2 M_{1}$; then

$$
\sum_{\xi \in \mathbb{F}_{p}}|\widehat{\varphi}(\xi)|\left|\sum_{\substack{0 \leq y<M_{0} \\ x \in I}} e_{p}(\xi(2 x-s)(a s+2 a y+b))\right| \gtrsim \frac{M^{3}}{2 M_{1}} .
$$

Next, for $z \in \mathbb{F}_{p}$, denote

$$
\eta(z)=\left|\left\{(x, y) \in I \times\left[1, M_{0}\right]:(2 x-s)(2 a y+b+a s) \equiv z(\bmod p)\right\}\right|,
$$

and write the left hand side of (1.7) as

$$
\begin{aligned}
& \sum_{\xi \in \mathbb{F}_{p}}|\widehat{\varphi}(\xi)|\left|\sum_{z \in \mathbb{F}_{p}} \eta(z) e_{p}(\xi z)\right| \\
\leq & \left(\sum_{\xi \in \mathbb{F}_{p}}|\widehat{\varphi}(\xi)|^{2}\right)^{1 / 2}\left(\sum_{\xi \in \mathbb{F}_{p}}\left|\sum_{z \in \mathbb{F}_{p}} \eta(z) e_{p}(\xi z)\right|^{2}\right)^{1 / 2} \\
= & \left(\frac{1}{p} \sum_{x \in \mathbb{F}_{p}}|\varphi(x)|^{2}\right)^{1 / 2} \sqrt{p}\left(\sum_{z \in \mathbb{F}_{p}} \eta(z)^{2}\right)^{1 / 2} \\
< & 2 M_{1}^{1 / 2}\left(\sum_{z \in \mathbb{F}_{p}} \eta(z)^{2}\right)^{1 / 2},
\end{aligned}
$$

by Cauchy-Schwarz and Parseval.

Recall that $(a, p)=1$. Let $I^{\prime}=I-\frac{s}{2}, I^{\prime \prime}=\left[1, M_{0}\right]+\frac{b+a s}{2 a} \subset \mathbb{F}_{p}$ so that

$$
\sum_{z \in \mathbb{F}_{p}} \eta(z)^{2}=E\left(I^{\prime}, I^{\prime \prime}\right)
$$

the multiplicative energy of $I^{\prime}$ and $I^{\prime \prime}$.

It is well-known that

$$
\begin{aligned}
E\left(I^{\prime}, I^{\prime \prime}\right) & \leq \log p \max \left\{\left|I^{\prime}\right|\left|I^{\prime \prime}\right|, \frac{\left|I^{\prime}\right|^{2}\left|I^{\prime \prime}\right|^{2}}{p}\right\} \\
& \leq \log p \max \left\{M_{1} M, \frac{M_{1}^{2} M^{2}}{p}\right\} .
\end{aligned}
$$

Thus, by (1.7), (1.9) and (1.10),

$$
\frac{M^{3}}{M_{1}} \lesssim M_{1}^{1 / 2}(\log p)^{1 / 2} \max \left\{M_{1}^{1 / 2} M^{1 / 2}, \frac{M_{1} M}{p^{1 / 2}}\right\} .
$$

Distinguish the cases $M_{1} M \leq p$ and $M_{1} M>p$, and then (1.11) implies

$$
M_{1} \gtrsim \min \left\{(\log p)^{-1 / 4} M^{5 / 4},(\log p)^{-1 / 5} M^{4 / 5} p^{1 / 5}\right\} .
$$




\section{Partial orbits of SMall diameters}

For $M<p^{c_{0}}$, one obtains the following stronger result. (Notation is as in Proposition [1)

Proposition 2. There exists $c_{0}>0$ such that

$$
\operatorname{diam} \mathcal{O}_{M}>\min \left(p^{c_{0}}, M^{\frac{1}{13} \log \log M}\right) .
$$

Consequently,

$$
\operatorname{diam} \mathcal{O}_{M} \gtrsim \min \left\{M p^{\frac{c_{0}}{5}}, \frac{1}{\log p} M^{\frac{4}{5}} p^{\frac{1}{5}}, M^{\frac{1}{13} \log \log M}\right\} .
$$

Proof. Let $\mathcal{O}_{M}=\left\{x_{0}, x_{1}, \ldots, x_{M-1}\right\}$ with $x_{j}=f\left(x_{j-1}\right)$ as before, and let diam $\mathcal{O}_{M}=M_{1}$. Since $\left|x_{j}-x_{0}\right| \leq M_{1}$, we can write $x_{j}=x_{0}+z_{j}$ with $z_{j} \in\left[-M_{1}, M_{1}\right]$. Thus, $a, b, c, x_{0}$ satisfy the $M-1$ equations

$$
a\left(x_{0}+z_{j}\right)^{2}+b\left(x_{0}+z_{j}\right)+c \equiv x_{0}+z_{j+1}(\bmod p), \quad j=0, \ldots, M-2,
$$

and the $\mathbb{F}_{p}$-variety

$$
\mathcal{V}_{p}=\bigcap_{j=0}^{M-2}\left[\left(r+z_{j}\right)^{2}+v\left(r+z_{j}\right)+w=u\left(r+z_{j+1}\right)(\bmod p)\right.
$$

in the variables $(u, v, w, r) \in \mathbb{F}_{p}^{4}$ is therefore nonempty. Note that the coefficients of the $M-1$ defining polynomials in $\mathbb{Z}[u, v, w, r]$ are $O\left(M_{1}^{2}\right)$.

Assume

$$
M_{1}<p^{c_{0}}
$$

with $c_{0}>0$ small enough. Elimination theory 2 implies that $\mathcal{V}_{p} \neq \emptyset$ is a $\mathbb{C}$-variety. Hence there are $U, V, W, R \in \mathbb{C}$ such that for all $j$,

$$
\left(R+z_{j}\right)^{2}+V\left(R+z_{j}\right)+W=U\left(R+z_{j+1}\right), \quad j=0, \ldots, M-2 .
$$

Obviously, $U \neq 0$, since $z_{1}, \ldots, z_{M-2}$ are distinct. We therefore have a quadratic polynomial

$$
F(z):=\frac{1}{U}(R+z)^{2}+\frac{V}{U}(R+z)+\frac{W}{U}-R=: A z^{2}+B z+C,
$$

satisfying

$$
F\left(z_{j}\right)=z_{j+1} \quad \text { in } \mathbb{C}, \quad \text { for } j=0, \ldots, M-2 .
$$

Since $z_{0}=0$, (2.4) and (2.5) imply $C=z_{1} \in \mathbb{Z} \cap\left[-M_{1}, M_{1}\right]$, and the equations

$$
\begin{aligned}
& z_{1}^{2} A+z_{1} B=z_{2}-z_{1}, \\
& z_{2}^{2} A+z_{2} B=z_{3}-z_{1}
\end{aligned}
$$

imply $A, B \in \mathbb{Q}$ with $A=\frac{a}{d}, B=\frac{b}{d}$, and $a, b, d \in \mathbb{Z}$ being $O\left(M_{1}^{3}\right)$. Equation (2.5) becomes

$$
z_{j+1}=\frac{a}{d} z_{j}^{2}+\frac{b}{d} z_{j}+C
$$

\footnotetext{
${ }^{2}$ See $[$ ], where a similar elimination procedure was used in a combinatorial problem. In particular, see C], Lemma 2.14 and its proof.
} 
Hence

$$
\frac{a}{d} z_{j+1}+\frac{b}{2 d}=\left(\frac{a}{d} z_{j}+\frac{b}{2 d}\right)^{2}+C \frac{a}{d}-\frac{b^{2}}{4 d^{2}}+\frac{b}{2 d} .
$$

Putting

and

$$
y_{j}=\frac{a}{d} z_{j}+\frac{b}{2 d} \in \frac{1}{2 d} \mathbb{Z}, \quad j=0, \ldots, M-1,
$$

$$
\frac{r}{s}=C \frac{a}{d}-\frac{b^{2}}{4 d^{2}}+\frac{b}{2 d} \quad \text { with } s>0,(r, s)=1,|r|, s=O\left(M_{1}^{6}\right),
$$

gives

$$
y_{j+1}=y_{j}^{2}+\frac{r}{s}, \quad j=0, \ldots, M-2 .
$$

Next, write $y_{j}=\alpha_{j} / \beta_{j}$, where $\beta_{j} \mid 2 d$ and $\left(\alpha_{j}, \beta_{j}\right)=1$; thus (2.7) gives

$$
\frac{\alpha_{j+1}}{\beta_{j+1}}=\frac{\alpha_{j}^{2}}{\beta_{j}^{2}}+\frac{r}{s}, \quad j=0, \ldots, M-2 .
$$

Note also that

$$
\left|\alpha_{j}\right|=O\left(M_{1}^{4}\right)
$$

Write the prime factorizations

$$
s=\prod_{p} p^{v(p)} \quad \text { and } \quad \beta_{j}=\prod_{p} p^{v_{j}(p)}, \quad j=0, \ldots, M-1 .
$$

Claim. $2 v_{j}(p) \leq v(p)$, for $j<M-O\left(\log \log M_{1}\right)$.

Proof. We may assume $v_{j}(p)>0$.

Case 1. $2 v_{j}(p)>v_{j+1}(p)$.

Fact 2.1 (which will be stated at the end of this section) and (2.8) imply that $v(p)=2 v_{j}(p)$.

Case 2. $2 v_{j}(p) \leq v_{j+1}(p)$.

Again, we separate two cases.

Case 2.1. $2 v_{j+1}(p)>v_{j+2}(p)$. Reasoning as in Case 1 , we have

$$
v(p)=2 v_{j+1}(p) \geq 2^{2} v_{j}(p)>2 v_{j}(p) .
$$

Case 2.2. $2 v_{j+1}(p) \leq v_{j+2}(p)$. Therefore, $v_{j+2}(p) \geq 2^{2} v_{j}(p)$. We repeat the argument for Case 2 with $j=j+1$. Continuing this process, after $\tau$ steps, we obtain either $v(p) \geq 2 v_{j}(p)$ or

$$
v_{j+\tau}(p) \geq 2^{\tau} v_{j}(p),
$$

when necessarily $\tau \lesssim \log v_{j+\tau}(p) \lesssim \log \log \beta_{j+\tau} \lesssim \log \log d \lesssim \log \log M_{1}$. Since $j+\tau \leq M$, the claim is proved.

It follows from the claim that $\beta_{j}^{2} \mid s$ for $j<M-O\left(\log \log M_{1}\right)$. Back to (2.8), if $v(p)>2 v_{j}(p)$ for some $j<M-O\left(\log \log M_{1}\right)$, then $v_{j+1}(p)=v(p)$. This contradicts the fact that $\beta_{j+1}^{2} \mid s$. So we conclude that

$$
\beta_{j}^{2}=s=: s_{1}^{2} \quad \text { for } j<M-O\left(\log \log M_{1}\right) .
$$


Hence

$$
\alpha_{j+1}=\frac{\alpha_{j}^{2}}{s_{1}}+\frac{r}{s_{1}}
$$

which implies

$$
\alpha_{j}^{2}+r \equiv 0 \quad \bmod s_{1} .
$$

Let $s_{1}=\prod p_{i}^{v_{i}}$. Then $\alpha_{j}$ satisfies (2.13) if and only if $\alpha_{j}$ satisfies $\alpha_{j}^{2}+r \equiv 0$ $\bmod p_{i}^{v_{i}}$ for all $i$. Since $-r$ is a quadratic residue modulo $p^{v}$ if and only if it is a quadratic residue modulo $p$ for odd prime $p$, we have

$$
\left|\left\{\pi_{s_{1}}\left(\alpha_{j}\right)\right\}_{j}\right| \leq 2 \cdot 2^{\omega\left(s_{1}\right)}<e^{\frac{\log s_{1}}{\log \log s_{1}}}<e^{\frac{4 \log M_{1}}{\log \log M_{1}}} .
$$

Here $\pi_{s_{1}}\left(\alpha_{j}\right)$ is the projection of $\alpha_{j}$ in $\mathbb{Z}_{s_{1}}$.

To show $M_{1}>M^{\frac{1}{13}} \log \log M$, we assume

$$
\log M_{1}<\frac{1}{13} \log M \log \log M .
$$

Then (2.14) implies there exists $\xi \in \mathbb{Z}_{s_{1}}$ such that

$$
|\mathcal{J}|=\left|\left\{0 \leq j \leq \frac{M}{2}: \pi_{s_{1}}\left(\alpha_{j}\right)=\xi\right\}\right|>M^{1 / 2} .
$$

Thus

$$
\alpha_{j_{1}}-\alpha_{j_{2}} \in s_{1} \mathbb{Z}, \quad \text { for } j_{1}, j_{2} \in \mathcal{J}
$$

and

$$
\left|\alpha_{j_{1}}-\alpha_{j_{2}}\right| \geq s_{1}, \quad \text { for } j_{1} \neq j_{2} \in \mathcal{J}
$$

In particular, there exists $j \in \mathcal{J}$ such that

$$
\left|\alpha_{j}\right| \geq \frac{M^{1 / 2}}{8} s_{1} \quad \text { and }\left.\quad|| \alpha_{j}|-| r\right|^{1 / 2} \mid>\frac{M^{1 / 2}}{8} s_{1} .
$$

Claim. Either $\left|\alpha_{j}\right|>10|r|^{1 / 2}$ or $\left|\alpha_{j+1}\right|>10|r|^{1 / 2}$.

Proof. Assume

$$
\left|\alpha_{j}\right|,\left|\alpha_{j+1}\right|<10|r|^{1 / 2} .
$$

Hence, $|r|^{1 / 2} \gtrsim M^{1 / 2} s_{1}$ by (2.17). From (2.12), (2.17) and (2.18),

$$
\begin{aligned}
10|r|^{1 / 2} s_{1}>\left|\alpha_{j+1}\right| s_{1} & =\left|\alpha_{j}^{2}+r\right| \\
& \geq\left(\left|\alpha_{j}\right|+|r|^{1 / 2}\right)\left(\left|\alpha_{j}\right|-|r|^{1 / 2}\right) \\
& \geq|r|^{1 / 2} \cdot \frac{M^{1 / 2}}{8} s_{1}
\end{aligned}
$$

a contradiction, proving the claim.

Thus, there exists $j<M / 2$ such that either

$$
\left|\alpha_{j}\right|>10 s_{1} \text { and }\left|\alpha_{j}\right|>10|r|^{1 / 2}
$$

or

$$
\left|\alpha_{j}\right|>10 s_{1} \text { and }\left|\alpha_{j+1}\right|>10|r|^{2} .
$$

Clearly, (2.19) implies (2.20). Indeed, by (2.12),

$$
\left|\alpha_{j+1}\right| \geq \frac{1}{s_{1}}\left|\alpha_{j}^{2}-\right| r|| \geq \frac{99}{100 s_{1}} \alpha_{j}^{2}>2\left|\alpha_{j}\right| .
$$


Iteration shows that

$$
\left|\alpha_{j+\frac{M}{3}}\right|>2^{\frac{M}{3}}\left|\alpha_{j}\right|>2^{\frac{M}{3}},
$$

contradicting (2.9). This proves (2.1).

Combining Proposition 1 and (2.1), we have (2.2).

Fact 2.1. Let $\frac{a_{1}}{d_{1}}, \frac{a_{2}}{d_{2}}, \frac{a_{3}}{d_{3}} \in \mathbb{Q}$ be rational numbers in lowest terms, and $p^{v_{p}\left(d_{i}\right)} \| d_{i}$. If $\frac{a_{1}}{d_{1}}+\frac{a_{2}}{d_{2}}+\frac{a_{3}}{d_{3}}=0$ and $v_{p}\left(d_{1}\right) \geq v_{p}\left(d_{2}\right) \geq v_{p}\left(d_{3}\right)$, then $v_{p}\left(d_{1}\right)=v_{p}\left(d_{2}\right)$.

\section{Full CyCles}

In this section, we will prove Theorem 2

Assume $M_{1}=\operatorname{diam} \mathcal{C}<p^{c_{0}}$ with $c_{0}$ as in Proposition 2, The proof of Proposition 2 gives a quadratic polynomial (cf. (2.7))

$$
F(z)=z^{2}+\frac{r}{s} \quad \text { with } r, s \in \mathbb{Z},|s|=O\left(M_{1}^{6}\right)
$$

and a rational $F$-cycle $\left\{y_{j}\right\}_{0 \leq j<T}$, i.e.

$$
y_{j+1}=F\left(y_{j}\right) \quad \text { for } 0 \leq j \leq T-2
$$

and $F\left(y_{T-1}\right)=y_{0}$.

We now invoke a result of $\mathrm{R}$. Benedetto [B], which gives quantitative bounds on the number of preperiodic points of a polynomial $f$ in a number field $(z$ is preperiodic if the set $\{z, f(z), f(f(z)), \ldots\}$ is finite). According to Theorem 7.1 in [B], the number of preperiodic points of $F$ in $\mathbb{Q}$ is bounded by

$$
(2 \sigma+1)\left[\log _{2}(2 \sigma+1)+\log _{2}\left(\log _{2}(2 \sigma+1)-1\right)+2\right]
$$

with $\sigma$ the number of primes where $F$ has bad reduction. Hence $\sigma \leq \omega(s) \leq$ $\frac{\log M_{1}}{\log \log M_{1}}$ and (3.2) implies

$$
T<4 \log M_{1}=4 \log \operatorname{diam} \mathcal{C} .
$$

\section{ACKNOWLEDGEMENTS}

The author would like to thank the referee for many helpful comments and the mathematics department of the University of California at Berkeley for its hospitality.

\section{REFERENCES}

[B] Robert L. Benedetto, Preperiodic points of polynomials over global fields, J. Reine Angew. Math. 608 (2007), 123-153, DOI 10.1515/CRELLE.2007.055. MR2339471 (2008j:11071)

[CCGHSZ] M.-C. Chang, J. Cilleruelo, M. Garaev, J. Hernandez, I. Shparlinski, A. Zumalacarregui, Points on curves in small boxes and applications (preprint).

[C] M. Chang, Factorization in generalized arithmetic progressions and applications to the Erdös-Szemerédi sum-product problems, Geom. Funct. Anal. 13 (2003), no. 4, 720-736, DOI 10.1007/s00039-003-0428-5. MR.2006555 (2004g:11007)

[CGOS] Javier Cilleruelo, Moubariz Z. Garaev, Alina Ostafe, and Igor E. Shparlinski, On the concentration of points of polynomial maps and applications, Math. Z. 272 (2012), no. 3-4, 825-837, DOI 10.1007/s00209-011-0959-7. MR2995141

[GS] Jaime Gutierrez and Igor E. Shparlinski, Expansion of orbits of some dynamical systems over finite fields, Bull. Aust. Math. Soc. 82 (2010), no. 2, 232-239, DOI 10.1017/S0004972709001270. MR2685147(2011j:11153)

Department of Mathematics, University of California, Riverside, California 92521

E-mail address: mcc@math.ucr.edu 